\title{
Characteristics of Disease Profile of Hospitalized Patients Referred to the Department of Cardiology in a Tertiary Care Hospital
}

\author{
Alam MZ1 ${ }^{1}$, Hossain $\mathrm{MS}^{2}$, Basit $\mathrm{MA}^{3}$, Hoque SJ4 , Mohibullah $\mathrm{AKM}^{5}$
}

\begin{abstract}
:
Objectives: Cardiology consultation for hospitalized patients is a common and sometimes mandatory practice in every country. This is because of increased morbidity and mortality of cardiac cases that also has other co-morbidities. We conducted this study to know the pattern of disease profile and the idea of requesting doctors about the cardiac diseases from the cases they referred to the department of Cardiology.

Methods: This prospective observational study was carried out in BIRDEM General Hospital, Shahbag, Dhaka from July to December 2017. We followed every case till the final and confirmed diagnoses were made. All the relevant collected data were compiled on a master data sheet. All findings were expressed as frequency with percentage and analysis were done using SPSS for windows version 22.0.
\end{abstract}

Results: This study revealed that majority of the referred cases to the department of cardiology was routine $(84.4 \%)$ and non-cardiac (57\%). Non-cardiac cases were referred mainly for the fitness of general anesthesia (GA) $(18.4 \%)$. But among the critical cases (14.0\%), mostly were cardiac $(9.1 \%)$. Among all the cardiac cases, Hypertension (8.8\%) got the highest referral followed by Acute Myocardial Infarction (AMI) (3.8\%) and Dilated Cardiomyopathy (DCM) (3.8\%).

Conclusions: Hospitalized patients were referred to the department of Cardiology differs according to the requesting unit. The surgical units referred their cases to get the cardiac clearance for a major surgery and the medical units referred for typical cardiac emergencies like AMI.

Key words: Cardiology, Referral, Hospitalized patients
Introduction:

It is a very common practice in medical science to refer a patient to a specialist for the purpose of better as well as

1. Associate professor, Department of Cardiology, BIRDEM General Hospital, Shahbag, Dhaka, Bangladesh.

2. Registrar, Department of Cardiology, BIRDEM General Hospital, Shahbag, Dhaka, Bangladesh.

3. Senior Medical Officer, Department of Cardiology, BIRDEM General Hospital, Shahbag, Dhaka, Bangladesh.

4. Assistant Professor, Department of Cardiology, BIRDEM General Hospital, Shahbag, Dhaka, Bangladesh.

5. Professor, Department of Cardiology, BIRDEM General Hospital, Shahbag, Dhaka, Bangladesh.

Address of Correspondence: Md Zahid Alam, Associate Professor, Department of Cardiology, BIRDEM General Hospital, Shahbag, Dhaka, Email: ilazybear@yahoo.com, Mobile: +8801711406290 more specific treatment. Not only complicated or undiagnosed cases are referred but also for taking a better second opinion is considered. Even hypertension is well managed in a primary care setting; still there are many referrals to the cardiologists for uncontrolled one. ${ }^{1}$ For hospitalized patients with multiple co-morbidities may need referral to more than one specialist care. In many hospitalized cases atypical presentations (eg in older age, women, diabetes mellitus) of a serious disease may causes aggravation of the primary disease for delayed referral. ${ }^{2-4}$

Internationally there are many recommendations of different institutions and bodies about how and when to refer a patient cardiology unit. For example, in Qeensland, 
before referring a patient to the cardiologist a confirm diagnosis of cardiac disease has to be made clinically as well as by relevant investigations. ${ }^{5}$ But it is more or less common in every recommendation about urgent referral which includes acute coronary syndrome (ACS), acute left ventricular failure (LVF), fatal arrhythmias (eg, ventricular tachycardia, complete heart block, etc). ${ }^{5-7}$

BIRDEM General Hospital is one of the largest tertiary care hospital in the center of the capital of Bangladesh which deals mainly with diabetic patients. As the diabetes mellitus is a multi-faced chronic disease, most of our patients have multiple co-morbidities. Thus it is natural that they need a team work for proper management. We conduct a trial of cross sectional for six months to see the disease profile of patients referred to the department of cardiology from different medical, surgical and gynecological units as well as to observe the idea of non-cardiologist about cardiac diseases. This type of referrals is given from all units on daily basis for mainly excluding any serious cardiac problems that may mimic other medical problems.

\section{Materials and Methods:}

This is a prospective observational study carried out, in BIRDEM General Hospital a tertiary care hospital, from July to December 2017. Our main objective was to see which condition of the patient compelled a doctor to take suggestion from the cardiology department. We excluded patients who were below 18 years of age, known psychiatric patients, and whose diagnoses were not confirmed during hospital stay. After excluding these cases, total number of patients referred to our unit was 668. We followed every case till the final and confirmed diagnoses were made. After confirming the diagnoses we categorized the patients into following groups: Referring unit, types of referral (urgent and routine), condition of the patients (asymptomatic, symptomatic but stable, symptomatic and critical), and cardiac or noncardiac cases. By urgent referral, the referring unit commonly indicates symptomatic and/or critical cases need prompt management. Many routine cases were asymptomatic and they were referred to us mainly for taking cardiac fitness for giving general anesthesia (GA). All the relevant collected data were compiled on a master data sheet. Data entry and analysis were done using SPSS for windows version 22.0. Frequency and percentage were calculated to find out the proportion of the findings.
Results:

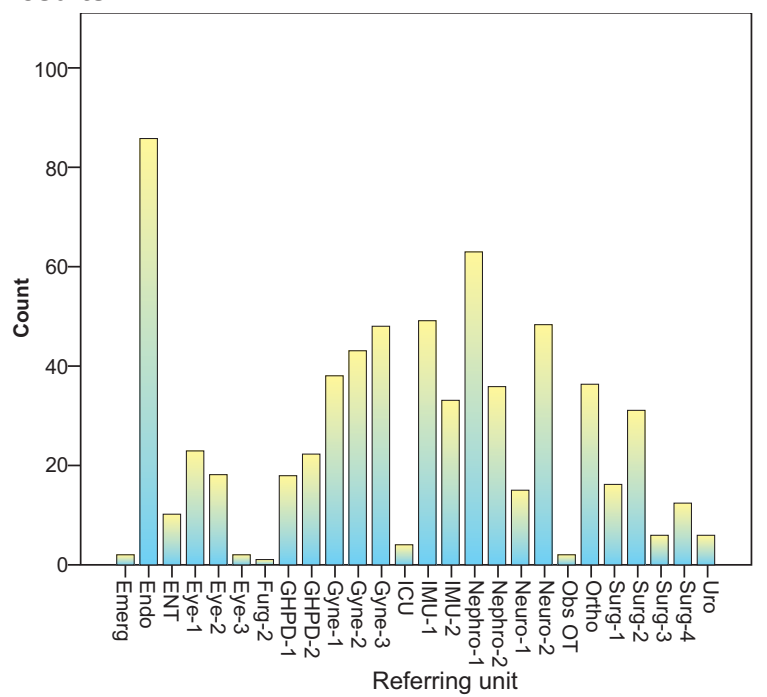

Fig.-1: Referring units and frequency of their referral

Total referring unit were 26 and total number of patients referred was 668 . The highest referring unit was Endocrinology $(n=86,12.8 \%)$, and the second highest unit was Nephrology unit-I ( $n=62,9.0 \%)$ (Figure: 1).

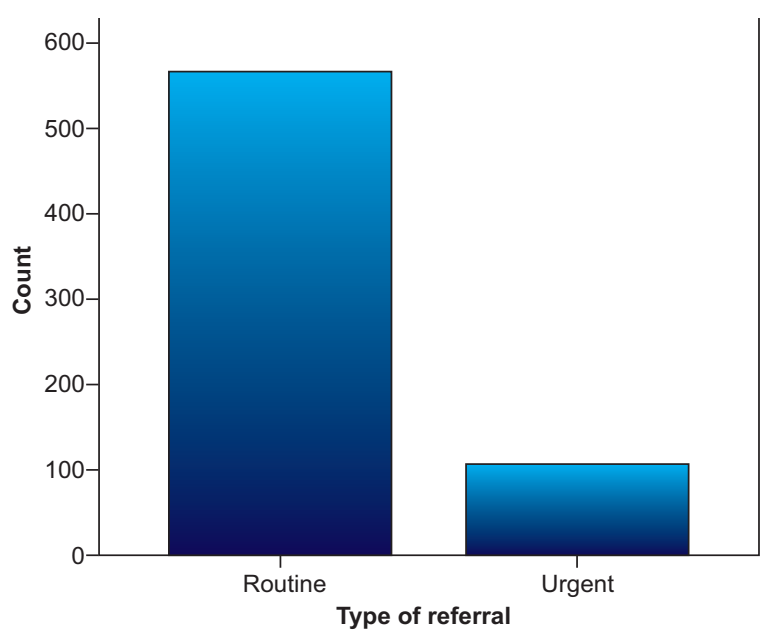

Fig.-2: Frequency of routine and urgent referral

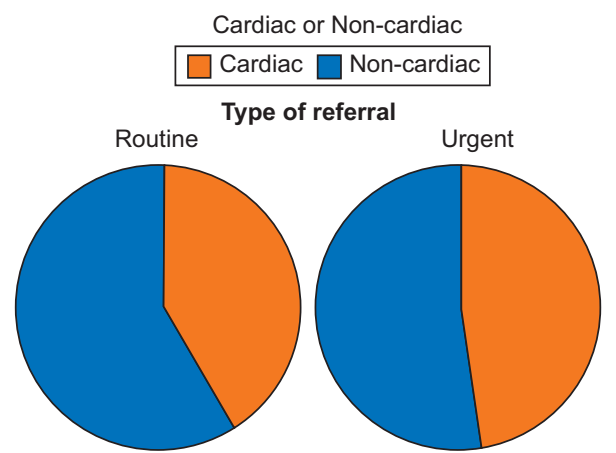

Fig.-3: Proportion of cardiac and non-cardiac cases in both routine and urgent referral 


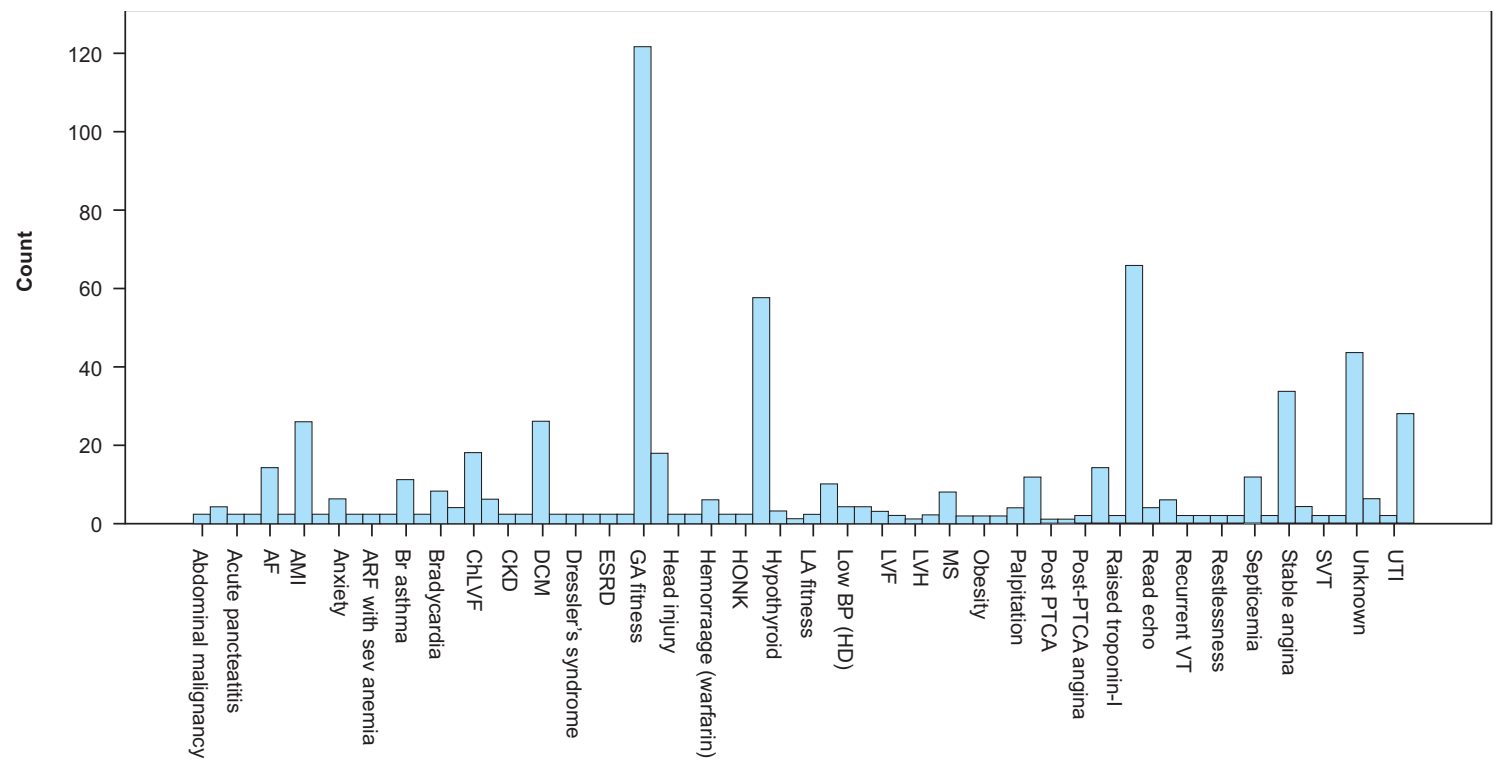

Fig.-4: Causes of referral

Most of the total referred cases were routine $(n=564$, $84.4 \%$ ). Among all the referral, more than half were diagnosed to have non-cardiac illness ( $n=382,57 \%$ ), or at least cardiac status was stable (Figure: 2 and 3 ).

Total number \& percentage of critical patients ( $n=94$, $14.0 \%)$ were mostly cardiac $(n=61,9.1 \%)$. Most of them were $\mathrm{AMI}$ and DCM with left ventricular failure (LVF).

The top ten causes of referral were as follows: Fitness for general anesthesia (GA) $(n=123,18.4 \%)$, interpreting ECG ( $n=66,9.8 \%)$, hypertension $(n=59,8.8 \%)$, routine cardiac consultation $(n=44,6.5 \%)$, stable angina $(=35$, $5.2 \%$ ), severe left ventricular (LV) systolic dysfunction $(n=28,4.1 \%)$, AMI $(n=26,3.8 \%)$, DCM $(n=26,3.8 \%)$, chronic LVF $(n=18,2.6 \%)$, atrial fibrillation (AF) $(n=14$, $2.0 \%)$.

Most critical cardiac cases were referred by Surgery unit2 (6 cases of AMI), Endocrinology unit (6 cases of AMI), and Nephrology units (12 cases of DCM).

\section{Discussion:}

Taking cardiology consultation from a non-cardiac case is common in every hospital. ${ }^{8}$ This is because of not only for excluding any cardiac diseases but also to make sure that no serious cardiac disease of atypical presentation is missed which can be fatal for the patients. ${ }^{2-4,9} \mathrm{We}$ conducted this cross sectional study with the main purpose of making a disease profile of the patients.

Majority of the referred cases were routine (84.4\%) and patients were asymptomatic with stable clinical condition.
Among all the referrals, numbers of cardiac cases were $286(42.8 \%)$ and non-cardiac cases were $382(57.1 \%)$. Increased number of non-cardiac cases is likely due to avoid any case fatality because atypical presentation of cardiac cases in elderly and diabetic patients are very common world wide $2,3,9$, and specially, diagnosis of chest pain of various reason may need to identify first in any such cases. ${ }^{9}$ A large number of cases $(6.5 \%)$ were referred to us without asking for any cardiac management, and these referred cases were also asymptomatic. Though we categorize these cases as "unknown" cause of referral, it is evident that taking a routine cardiac consultation of a diabetic patient in the form of "routine check-up" was the main reason. This is also the same cause of referral to the cardiology unit in abroad. ${ }^{8}$

As a universal rule, the frequency of taking cardiology consultation before going for surgery (esp under GA) is much higher than from other medical speciality. ${ }^{8}$ This is why, in our study also reflects this pattern of referral. Interpreting ECG is an interesting cause of referral, because all of these patients were completely asymptomatic and their ECG findings were also normal. We think, therefore, these were also a part of routine cardiac check-up. Some non-cardiac cases drew our attention. These were End Stage Renal Disease (ESRD), sepsis, Multi Organ Dysfunction Syndrome (MODS), headache, pneumonia, hypothyroidism, stroke, bronchial asthma, etc. Because, though they were primarily noncardiac, they could, either, affect the cardiovascular 
system during the course of the disease, or might be the effect of some cardiac drugs (eg, nitrate, beta blocker). Interestingly, a good number of critical cardiac cases were initially admitted in Surgical unit and nephrology units for their atypical presentations. Six cases of AMI were admitted in Surgical unit with upper abdominal pain and vomiting, and 12 cases of DCM were admitted in Nephrology units with generalized body swelling with nausea. It is common for the diabetic patients with ACS to present with atypical symptoms. ${ }^{3}$

Most surprising causes of referral, though insignificant in number, were urinary tract infection (UTI) $(n=2,0.3 \%)$, peripheral neuropathy $(n=2,0.3 \%)$, acute pancreatitis $(n=1,0.1 \%)$, aphasia $(n=1,0.1 \%)$, fitness for local anesthesia $(n=1,0.1 \%)$, and pain in the eye following eye surgery $(n=1,0.1 \%)$. We could not find any cardiac disease in any of these patients during their hospital stay.

This study had some limitations. First, this was an observational study. Analytical study may reveal some association that may alter the management plan. Second, the absence of demography of the patients, which, if present, may further enrich the disease profile. Third, this was a single center study and the requesting doctors were not asked about the cause of referral in obscured cases. Fourth, no associated co-morbidities were included in our study. If these were included, not only the magnitude of the problems was clarified but also the prognosis of many cases (esp the critical ones) could be predicted.

There were several strength of our study. To our knowledge, this study was one of the largest studies in our country on hospitalized patients. We did not collected the referral data according to the requesting doctors, but we also followed every cases (where needed) to reach a confirmed diagnosis to avoid the referral bias. For example, few cases were referred for suspected arrhythmia. But after thorough evaluation, they were finally diagnosed to have anxiety neurosis. Moreover, referral were categorized as urgent, routine, critical, stable, cardiac, and non-cardiac to understand the magnitude of the problem, and also, the idea of the requesting doctors more clearly. We also meticulously searched the association between non-cardiac cases and possible cardiac involvement as described earlier.

This study can give an idea to both the cardiologist as well as other doctors about the pattern of referral. We emphasize the importance of non-cardiology doctors for all resident doctors to identify cardiac emergencies as well as non-cardiac critical conditions mimicking cardiac disease to avoid case fatalities. This, like other hospital, will distribute the workload equally among doctors. ${ }^{10}$

\section{References:}

1. McCormack T, Cappuccio FP. 10 steps before you refer for: hypertension. Br J Cardiol 2008;15:254-7.

2. Torbal A, Boersma E, Kors JA, Herpen GV, Deckers JW, Kuip DAMVD, et al. Incidence of recognized and unrecognized myocardial infarction in men and women aged 55 and older: the Rotterdam Study. Eur Heart J 2006;27:729-36.

3. Khafaji HARH, Suwaidi JMA. Atypical presentation of acute and chronic coronary artery disease in diabetics. World J Cardiol. 2014;6(8): 802-13.

4. Feldman DE, Xiao Y, Bernatsky S, Haggerty J, Leffondré $\mathrm{K}$, Tousignant $\mathrm{P}$, et al. Consultation with cardiologists for persons with new-onset chronic heart failure: A population-based study. Can J Cardiol. 2009;25(12): 690-4.

5. Referral to cardiology - Queensland health [Internet] Available from: http://www.health.qld.gov.au/_data/ assets/pdf_file/0030/435684/cf-ref-card.pdf

6. Referral guidelines: cardiology referral process: cardiology - Alfred Health [Internet] Available from: http://www.alfredhealth.org.au/contents/resources/ referral-guidelines/Cardiology-ReferralGuidelines.pdf

7. Cardiology referral recommendations [Internet] Available from: http://www.gp.health.wa.gov.au/ cpac/speciality/docs/refrec001.pdf

8. Marques AC, Calderaro D, Yu PC, Gualandro DM, Carmo GA, Azevedo FR, et al. Impact of cardiology referral: clinical outcomes and factors associated with physicians' adherence to recommendations. Clinics 2014;69(10):666-71.

9. El-Menyar A, Jubaid M, Sulaiman K, AlMahmeed W, Singh R, Alsheikh-Ali AA, et al. Atypical presentation of acute coronary syndrome: A significant independent predictor of in-hospital mortality. J Cardiol 2011;57(2):165-71.

10. Cronin E, Graham I. "When are you seeing my patient?"-an analysis of the cardiology consultation service in a teaching hospital. Ir Med J. 2010 May;103(5):144-6. 